\title{
Prática de atividade física em meio à pandemia da COVID-19: estudo de base populacional em cidade do sul do Brasil
}

\author{
Physical activity during the COVID-19 pandemic: \\ a population-based cross-sectional study in a city of South Brazil
}

Inácio Crochemore-Silva (https://orcid.org/0000-0001-5390-8360) ${ }^{1}$
Alan Goularte Knuth (https://orcid.org/0000-0002-2030-5747) ${ }^{2}$
Andrea Wendt (https://orcid.org/0000-0002-4640-2254) ${ }^{3}$
Bruno Pereira Nunes (https://orcid.org/0000-0002-4496-4122) ${ }^{4}$
Pedro Curi Hallal (https://orcid.org/0000-0003-1470-6461) ${ }^{1}$
Leonardo Pozza Santos (https://orcid.org/0000-0002-3993-3786) ${ }^{5}$
Jenifer Harter (https://orcid.org/0000-0002-9130-4290) ${ }^{6}$
Débora da Cruz Payão Pellegrini (https://orcid.org/0000-0002-4285-5643) ${ }^{7}$

${ }^{1}$ Programa de Pós-

Graduação em Educação

Física, Universidade Federal

de Pelotas (UFPEL). R.

Marechal Deodoro 1160,

Centro. 96020-220 Pelotas

RS Brasil.

inacio_cms@yahoo.com.br

${ }^{2}$ Programa de Pós-

Graduação em Saúde

Pública, FURG. Rio Grande

RS Brasil.

${ }^{3}$ Programa de Pós-

Graduação em

Epidemiologia, UFPEL.

Pelotas RS Brasil.

${ }^{4}$ Programa de Pós-

Graduação em Enfermagem,

UFPEL. Pelotas RS Brasil.

${ }^{5}$ Curso de Nutrição,

Universidade Federal do

Pampa (Unipampa). Itaqui

RS Brasil.

${ }^{6}$ Curso de Enfermagem,

Unipampa. Uruguaiana RS

Brasil.

${ }^{7}$ Curso de Medicina

Veterinária, Unipampa.

Uruguaiana RS Brasil.

\begin{abstract}
This study aimed to describe leisure-time physical activity (LPA) during the COVID-19 pandemic in a municipality of Rio Grande do Sul state, southern Brazil, according to gender, level of education, and adherence to social distancing. A population-based and cross-sectional descriptive study was carried out in Bagé (RS), Brazil. LPA during the pandemic, place of activity, and Physical Education professional's supervision, were described. The sample included 377 adults, and $24.4 \%$ reported LPA during the pandemic. Marked inequalities were observed. LPA prevalence among men was 20 percentage points ( $p p$ ) higher than women and $40 p p$ higher among those with higher schooling than those with lower schooling. Among those reporting LPA, $53.5 \%$ practiced at home, and $64.8 \%$ did not report Physical Education professional supervision. No differences were observed between LPA and level of social distancing. Besides the recurrent discourse that people should include physical activity in the pandemic context, in the light of the marked inequalities observed, this study addressed sociocultural aspects and emphasized that LPA promotion initiatives require humanized approaches that consider the unequal living conditions of Brazilians.

Key words Motor activity, Social distancing, Social determinants of health
\end{abstract}

Resumo O objetivo deste estudo foi descrever a prática de atividade física de lazer (AFL) em meio a pandemia do COVID-19 em cidade do Rio Grande do Sul, avaliando desigualdades entre os sexos e grupos de escolaridade e diferenças de acordo com o nivel de distanciamento social. Trata-se de um estudo descritivo de corte transversal e base populacional com adultos no município de Bagé. Foram descritas a prática de AFL durante a pandemia, local de prática e orientação profissional. Na amostra de 377 adultos, 24,4\% relataram prática de AFL durante a pandemia. Foram observadas marcantes desigualdades; a prevalência de AFL entre homens foi 20 pontos percentuais ( $p p$ ) maior do que entre as mulheres, e $40 p p$ maior no grupo de maior escolaridade comparado ao grupo de menor escolaridade. Entre os que praticaram AFL durante a pandemia, 53,5\% relataram a prática em casa e 64,8\% não teve auxílio de um profissional de Educação Física. Não houve diferença na AFL de acordo com níveis de distanciamento social. Para além da reprodução do discurso de que as pessoas devam praticar atividade física no contexto da pandemia, este estudo buscou discutir aspectos socioculturais, enfatizando, à luz das desigualdades observadas, que a promoção de AFL necessita de olhar humanizado e atento à vida desigual das pessoas no Brasil.

Palavras-chave Atividade motora, Isolamento social, Determinantes sociais de saúde 


\section{Introdução}

O novo Coronavírus (SARS-CoV-2) surgiu em dezembro de 2019, em uma província chinesa, e vem se espalhando pelo mundo com padrões sem precedentes. Em 11 de março de 2020 a Organização Mundial de Saúde considerou a COVID-19 (doença causada pelo SARS-CoV-2) uma pandemia ${ }^{1}$. Entre maio e junho de 2020, as Américas passaram a ser o epicentro da pandemia, local com maior número de casos novos da COVID-19. Com a ausência de tratamento específico e efetivo, bem como sem uma vacina para imunizar a população, medidas não farmacológicas como as relacionadas à higiene pessoal, uso de máscara e, principalmente, o distanciamento social, têm sido as melhores alternativas para diminuir a propagação da doença, evitando que os sistemas de saúde entrem em colapso ${ }^{2,3}$.

Neste contexto, atreladas às indicações de distanciamento social pelas autoridades de saúde competentes, diversas práticas sociais e de saúde foram discutidas e recomendadas no contexto da pandemia. Um dos discursos acionados de forma enfática foi a prática de atividade físi$\mathrm{ca}^{4-7}$, principalmente abordando seus potenciais benefícios relacionados à imunidade, doenças crônicas e saúde mental. Em consonância com o fato de que a maioria das pessoas estavam em seus domicílios, em situação de distanciamento social, um dos formatos recorrentes de atividade física estavam relacionadas a práticas mediadas por tecnologias, onde profissionais da área utilizaram redes e mídias sociais para comunicar sobre a importância desta, pois diversos locais de prática, como parques, praças e academias foram fechados no contexto da COVID-19.

Destaca-se que o discurso sobre a atividade física é, desde antes da pandemia, majoritariamente envolvido por uma demasiada carga de simplicidade e que deveria ser problematizado a partir de uma análise socialmente contextualizada à população brasileira. Dados oriundos de capitais brasileiras em 2019 apontam que 39\% dos adultos praticam atividade física de lazer conforme as recomendações de saúde ${ }^{8,9}$. Há ainda poucas políticas públicas que ofertam programas com práticas corporais e atividades físicas, tornando o acesso a um profissional dessa área restrito e acessível principalmente aos indivíduos de maior poder aquisitivo ${ }^{10,11}$.

No atual contexto da pandemia, esse cenário pode ser ampliado se observarmos, de forma bastante pragmática, a já relativamente baixa participação em atividade física antes da pandemia, os limitados espaços disponíveis nas residências, bem como o desigual acesso à internet para aulas on-line e/ou ainda o restrito contato com professores e profissionais de Educação Física. Além disso, à luz dos determinantes sociais da saúde, está o desafio de pautar a prática de atividade física frente às demandas de sobrevivência e garantia das condições de vida com a crise política/ financeira do país e com as limitadas políticas de mitigação dos problemas econômicos e sociais. Minayo e Freire ${ }^{12}$ destacam que a pandemia no contexto brasileiro exacerba as desigualdades em saúde, e fazem a analogia de que o país como um todo segue na tempestade, mas não necessariamente no mesmo barco.

De acordo com revisão da literatura, não foram encontrados estudos de base populacional tanto no Brasil quanto no mundo, não sendo conhecida a proporção da população que relata a prática de atividade física durante o período de distanciamento social. Assim, o objetivo do presente estudo foi descrever a prática de atividade física de lazer em meio a pandemia do COVID-19 em uma cidade do Rio Grande do Sul, avaliando diferenças relacionadas ao nível de distanciamento social e as desigualdades em termos de sexo e escolaridade.

\section{Métodos}

\section{Delineamento e população alvo}

Trata-se de um estudo de caráter descritivo com corte transversal e base populacional, realizado na cidade de Bagé, Rio Grande do Sul. As presentes análises fazem parte da primeira rodada de um estudo maior que busca avaliar ao longo de quatro inquéritos a prevalência de infecção por SARS-CoV-2 no município. Este estudo foi aprovado pelo Comitê de Ética em Pesquisa da Universidade Federal de Pelotas e foi desenvolvido pela Universidade Federal do Pampa (UNIPAMPA) em parceria com a Secretaria de Saúde do Município de Bagé e apoio da Universidade Federal de Pelotas. Embora inspirado e semelhante metodologicamente, este estudo não faz parte da iniciativa de nível estadual do estudo EPICOVID-RS ${ }^{13}$.

O município de Bagé se encontra na região sul do estado, possui cerca 120 mil habitantes, tem um Produto Interno Bruto (PIB) de R \$24.601,28 e Índice de Desenvolvimento Humano (IDH) de 0,740 , de acordo com estimativas do Instituto Brasileiro de Geografia e Estatística ${ }^{14}$. A coleta 
de dados ocorreu entre 7 e 9 de maio, quando no município havia pouco mais de 30 casos confirmados de COVID-19. Destaca-se que desde o dia 19 de março de 2020 havia sido decretado situação de calamidade pública no município (decreto municipal 050/2020), com a adoção de medidas rígidas de distanciamento social, permanecendo abertas apenas as atividades essenciais em vigor. No período em que foi realizada a coleta de dados, estavam vigentes os decretos 58 e 59/2020 (publicados em 15 e 30 de abril, respectivamente), os quais, entre outros aspectos, mantinham a suspensão de atividades da rede de ensino (pública e privada), clubes sociais e esportivos, academias de ginástica, cinemas e bares e estabeleciam horários para estabelecimentos comerciais para fins de atendimento/abastecimento mínimo da população (com medidas de higienização, utilização de equipamento de proteção individual - EPIs e quantidade limitada de clientes) (mais informações disponíveis em: www.bage.rs.gov.br).

\section{Processo de amostragem e coleta de dados}

Todos os moradores da zona urbana de Bagé foram considerados elegíveis para participar da pesquisa, embora os dados deste estudo se refiram a adultos com 18 anos de idade ou mais. $\mathrm{O}$ processo de amostragem foi realizado em múltiplos estágios. Inicialmente foram selecionados aleatoriamente 40 setores censitários dos 167 existentes em Bagé. Para a seleção dos setores foi utilizada a grade de setores censitários que seria utilizada pelo IBGE no Censo Demográfico de 2020. Posteriormente, foram selecionados aleatoriamente 10 domicílios por setor censitário, totalizando 400 domicílios visitados. Por fim, em cada residência foi sorteado aleatoriamente um indivíduo para a testagem sorológica e a resposta ao questionário.

A coleta de dados foi realizada pelos Agentes Comunitários de Saúde (ACS) e técnicos de enfermagem vinculados à rede de atenção básica de saúde do município, utilizando os devidos EPIs. Ao todo, 19 profissionais foram submetidos a um treinamento para aplicação do teste sorológico e aplicação dos questionários.

\section{Instrumentos de pesquisa}

O questionário de atividade física de lazer foi proposto pelos autores a partir da pandemia e apresentou duas abordagens. A primeira questionou sobre a prática de atividades físicas como ginásticas, esportes, caminhadas ou corri- das (entre outras) no período de lazer durante a pandemia da COVID-19 no contexto do distanciamento social. Em caso de respostas positivas, os entrevistados eram questionados sobre o local de prática (em casa/apartamento; ao ar livre em praças/pistas/ruas do bairro; ambientes fechados como academias), sobre o recebimento de orientação de profissionais de Educação Física e, em caso positivo, sua forma de contato (se continuou acompanhamento profissional anterior de forma on-line ou presencial; se iniciou acompanhamento profissional de forma on-line ou presencial ou se foi por meio de vídeos e postagens retirados da internet, mas sem vínculo com o profissional). A segunda parte do questionário buscou caracterizar a prática de atividade física de lazer na semana anterior à entrevista, aplicando uma versão adaptada do domínio de lazer do Questionário Internacional de Atividade Física (IPAQ - International Physical Acitivity Questionnaire), avaliando se a pessoa entrevistada havia praticado atividades físicas como ginásticas, esportes, caminhadas ou corridas no lazer durante os últimos sete dias e, em caso de resposta positiva, era averiguado em quantos dias e a média de tempo em cada dia. Neste componente do questionário foi estimado o percentual de participantes que atingiam as recomendações de 150 minutos semanais de atividade física ${ }^{15}$.

As variáveis sociodemográficas coletadas foram sexo (feminino; masculino) e escolaridade (nenhuma ou frequentou apenas o fundamental; frequentou médio ou superior incompleto; superior completo). Além disso, variáveis de distanciamento social também foram coletadas, seguindo o mesmo questionário do estudo EPICOVID-RS ${ }^{13}$. Os entrevistados foram questionados "com relação ao distanciamento social que estava sendo orientado pelas autoridades de saúde, ou seja, ficar em casa e evitar contato com outras pessoas", o quanto eles consideram que estavam conseguindo fazer (muito pouco; pouco; mais ou menos; bastante; praticamente isolado de todo mundo), e como estava sendo sua rotina de atividades (ficava em casa o tempo todo; saía apenas para coisas essenciais como comprar comida; saía de vez em quando para compras e esticar as pernas; saía todos os dias para alguma atividade; saía todos os dias, o dia todo, para trabalhar ou outra atividade regular).

\section{Análises de dados}

As principais análises apresentadas são descritivas, baseadas na apresentação de proporções 
e seus respectivos intervalos de confiança das variáveis categóricas. $\mathrm{O}$ teste de qui-quadrado de heterogeneidade e de tendência linear foi utilizado para testar diferenças estatisticamente significativas no desfecho de acordo com variáveis categóricas nominais e ordinais, respectivamente. Todas as análises foram realizadas no software Stata 16.0 , adotando $5 \%$ como nível de significância e levando em consideração o efeito de delineamento do estudo (comando svy).

\section{Resultados}

A amostra foi composta por 377 adultos, dos quais $37,1 \%$ eram do sexo masculino e $16,9 \%$ apresentavam nível superior completo. Com relação às variáveis relacionadas ao distanciamento social, $68,4 \%$ relataram bastante adesão às medidas vigentes ou estarem praticamente isolados e $74,3 \%$ disseram ficar sempre em casa ou apenas sair para compras essenciais (Tabela 1).

A prevalência de prática de atividade física de lazer durante a pandemia foi de $24,4 \%$. Quando avaliada a prática de atividade física de lazer na semana anterior à entrevista conforme a reco- mendação, 7,7\% dos participantes atingiram 150 minutos. Diferenças importantes foram identificadas de acordo com o sexo dos participantes com relação à prática de atividade física de lazer durante a pandemia $(\mathrm{p}<0,001)$ e à realização de 150 minutos na semana anterior à entrevista $(\mathrm{p}=$ $0,008)$. Destaca-se que enquanto $37,1 \%$ dos homens relataram a prática de atividade física de lazer durante a pandemia, entre as mulheres foi de $16,9 \%$, resultando em uma diferença de 20 pontos percentuais entre os dois grupos (Tabela 1).

A Figura 1 apresenta a prática de atividade física de lazer durante a pandemia na amostra geral e entre homens e mulheres de acordo com a escolaridade dos participantes. A prevalência de prática variou de 9,8\% entre os participantes com menor escolaridade a $50,9 \%$ entre os que relataram ter ensino superior completo $(\mathrm{p}<$ 0,001 ), uma diferença absoluta maior de 40 pontos percentuais entre os grupos (Figura 1).

O local de realização de atividade física de lazer durante a pandemia e se a prática teve auxílio de profissional de Educação Física são apresentados na Figura 2. Mais da metade dos entrevistados que praticaram atividade física relatou a casa

Tabela 1. Descrição da amostra. Bagé, RS ( $\mathrm{N}=377)$

\begin{tabular}{|c|c|c|c|c|c|c|}
\hline & \multicolumn{2}{|r|}{ Homens } & \multicolumn{2}{|r|}{ Mulheres } & \multicolumn{2}{|r|}{ Total } \\
\hline & $\mathbf{N}$ & $\%($ IC 95\%) & $\mathbf{N}$ & $\%($ IC 95\%) & $\mathbf{N}$ & $\%($ IC 95\%) \\
\hline Prática de AF de lazer ${ }^{\star}$ & 52 & $37,1(27,3-48,1)$ & 40 & $16,9(11,9-23,3)$ & 92 & $24,4(19,0-30,7)$ \\
\hline Prática AF de lazer (150 minutos) ${ }^{\star \star}$ & 18 & $12,9(7,6-21,0)$ & 11 & $4,6(2,3-9,1)$ & 29 & $7,7(4,9-11,9)$ \\
\hline \multicolumn{7}{|l|}{ Escolaridade $^{\star * *}$} \\
\hline $\begin{array}{l}\text { Nenhuma ou frequentou } \\
\text { fundamental }\end{array}$ & 44 & $37,6(27,5-49,0)$ & 99 & $46,9(39,0-54,7)$ & 143 & $43,6(36,5-51,0)$ \\
\hline $\begin{array}{l}\text { Frequentou médio ou superior } \\
\text { incompleto }\end{array}$ & 48 & $41,0(31,9-54,9)$ & 82 & $38,9(31,9-50,8)$ & 130 & $39,6(33,7-45,9)$ \\
\hline Superior completo & 25 & $21,4(13,3-32,4)$ & 30 & $14,2(8,5-22,7)$ & 55 & $16,8(10,8-25,2)$ \\
\hline \multicolumn{7}{|l|}{ Distanciamento social ${ }^{\star * *}$} \\
\hline Muito pouco & 13 & $9,4(5,2-16,5)$ & 13 & $5,5(2,5-11,9)$ & 26 & $7,0(4,1-11,5)$ \\
\hline Pouco & 10 & $7,2(4,1-12,6)$ & 14 & $5,9(3,1-11,0)$ & 24 & $6,4(3,9-10,5)$ \\
\hline Mais ou menos & 39 & $28,3(19,9-38,4)$ & 29 & $12,3(8,3-17,8)$ & 68 & $18,2(13,2-24,5)$ \\
\hline Bastante & 49 & $35,5(25,0-47,6)$ & 100 & $42,4(33,9-51,3)$ & 149 & $39,8(32,0-48,2)$ \\
\hline Praticamente isolado & 27 & $19,6(12,6-29,1)$ & 80 & $33,9(26,2-42,6)$ & 107 & $28,6(22,2-36,0)$ \\
\hline \multicolumn{7}{|l|}{ Rotina de atividades } \\
\hline Fica em casa & 29 & $20,7(15,0-27,9)$ & 73 & $30,8(24,1-38,4)$ & 102 & $27,1(22,0-32,7)$ \\
\hline Sai para compras essenciais & 58 & $41,4(32,4-51,1)$ & 120 & $50,6(44,3-57,0)$ & 178 & $47,2(41,4-53,0)$ \\
\hline Sai as vezes & 5 & $3,6(1,6-7,7)$ & 4 & $1,3(0,4-3,9)$ & 8 & $2,1(1,0-4,4)$ \\
\hline Sai todos os dias & 15 & $10,7(6,1-18,1)$ & 12 & $5,1(2,8-9,1)$ & 27 & $7,2(4,7-10,8)$ \\
\hline Sai todos os dias para trabalho & 33 & $23,6(16,2-32,9)$ & 29 & $12,2(7,7-18,9)$ & 62 & $16,4(11,9-22,2)$ \\
\hline
\end{tabular}




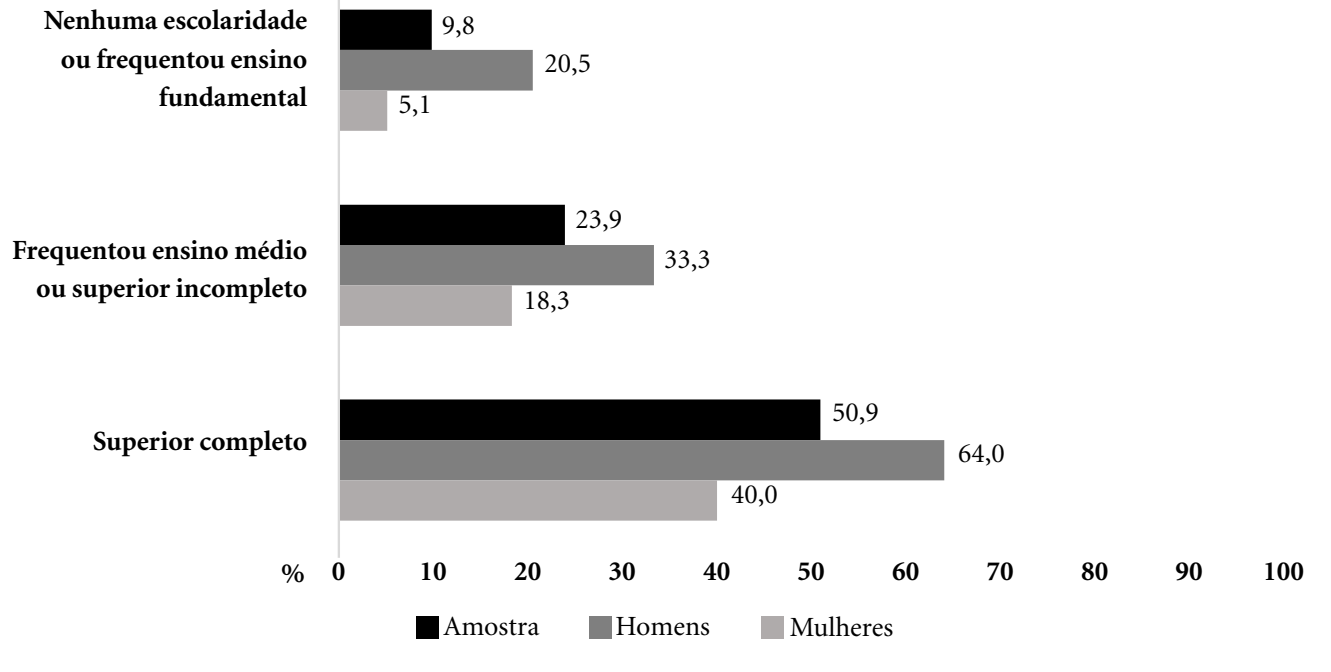

Figura 1. Prevalência de prática de atividade física de lazer durante a pandemia de acordo com sexo e escolaridade (diferença entre homens e mulheres e entre os grupos de escolaridade foram estatisticamente significativas; $\mathrm{p}<0,001)$. Bagé, RS $(\mathrm{N}=328)$.

(B) Atividade física com acompanhamento de profissional (\%)

(A) Local de prática de atividade física (\%)

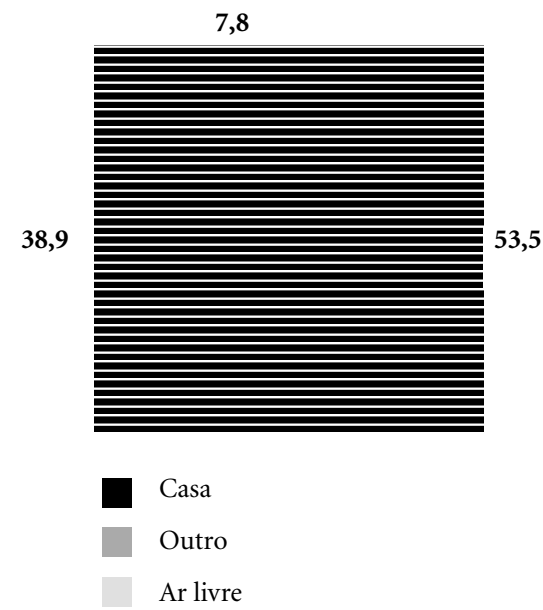

20,9

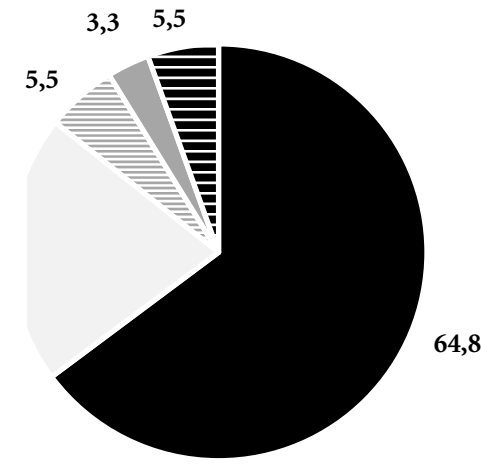

Sem ajuda de profissional

Iniciou acompanhamento

Outro

Continuou acompanhamento

Sem vínculo (ex: internet, reportagens)

Figura 2. Local de prática de atividade física de lazer (A) e auxílio de profissional de Educação Física (B) (Bagé, $\mathrm{N}=92$; indivíduos que relataram praticar atividade física de lazer durante a pandemia). 
como local de prática $(53,5 \%)$ e que não teve auxílio de um profissional (64,8\%). Também entre aqueles que realizaram atividade física de lazer, cerca de $20 \%$ relatou dar continuidade a prática de atividade física com auxílio profissional que tinha anteriormente à pandemia.

Quanto ao distanciamento social, os indivíduos que mais relataram prática de atividade física de lazer $(37,7 \%)$ foram aqueles do grupo intermediário com relação à adesão ao distanciamento (relataram estar "mais ou menos isolados"). Em ambos os grupos extremos dessa variável (muito pouco isolamento ou que se consideravam praticamente isolados) cerca de $20 \%$ relatou a prática de atividade física de lazer. Quanto à rotina de atividades dos entrevistados, houve um aumento da prevalência de prática de atividade física durante a pandemia de acordo com o aumento da frequência com a qual saiam de casa $(12,5 \%$ entre os que ficam em casa e
$40,7 \%$ entre os que saem todos os dias), exceto o grupo de pessoas que saiam todos os dias devido ao trabalho $(27 \%$ relataram prática de atividade física) (Figura 3).

\section{Discussão}

O presente estudo apresenta de forma pioneira no Brasil uma descrição da prática de atividade física de lazer em tempos de enfrentamento da pandemia de COVID-19 com base em dados com representatividade populacional. Cerca de um quarto dos participantes relataram a prática de atividades físicas como ginásticas, esportes, caminhadas ou corridas no seu lazer durante a pandemia, sendo majoritariamente realizada em casa e sem orientação de profissionais de Educação Física. Além disso, importantes desigualdades foram observadas desfavoráveis às mulheres $\mathrm{e}$
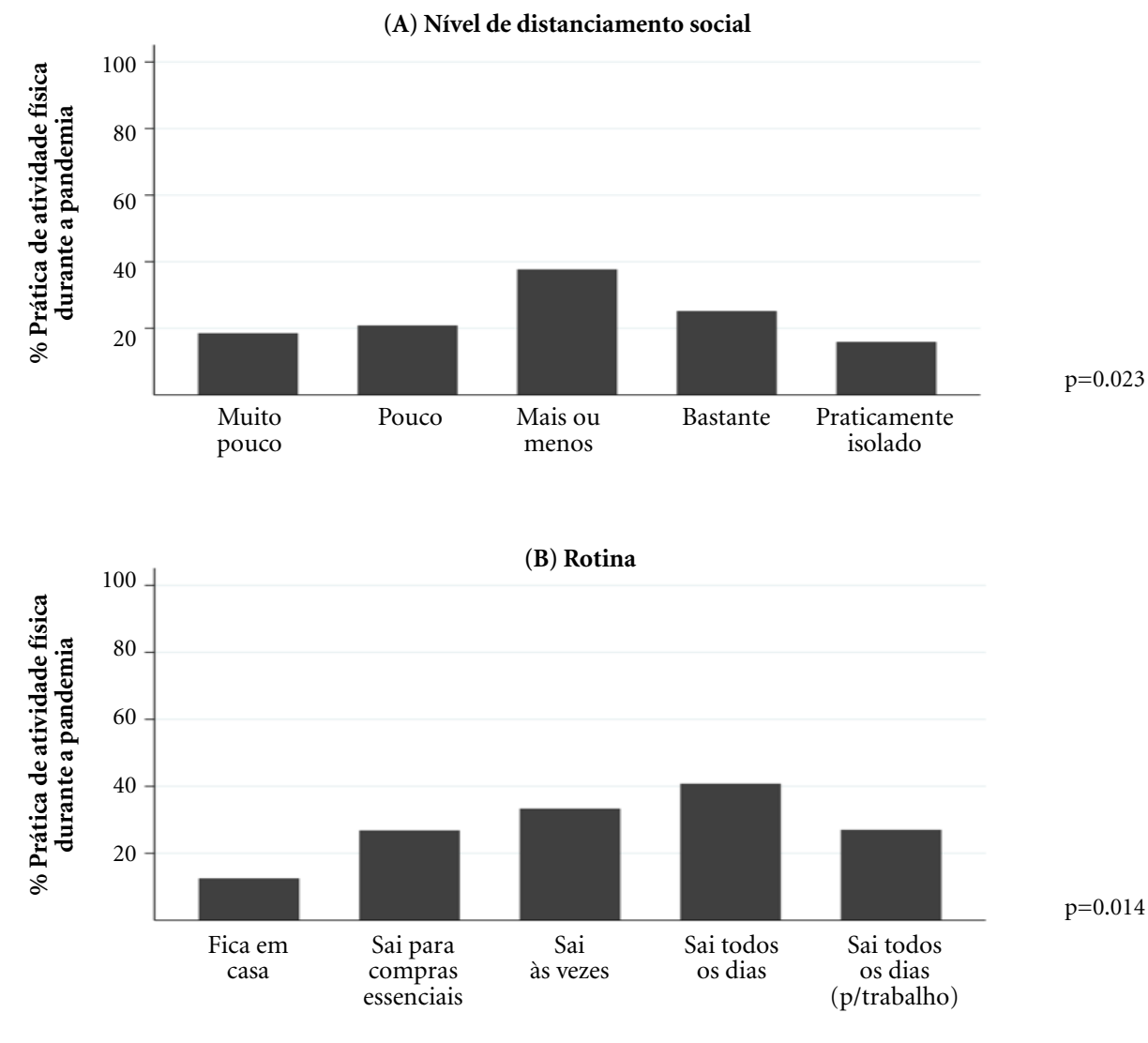

Figura 3. Relação entre prática de atividade física de lazer e distanciamento social (A) e rotina de atividades (B). Bagé, RS $(\mathrm{N}=382)$. 
aos menos escolarizados. Diferenças absolutas na prevalência de prática de atividade física de lazer durante a pandemia de 20 e 40 pontos percentuais foram descritas entre homens e mulheres e entre grupos de maior e menor escolaridade, respectivamente.

A comparação dos achados com a literatura apresenta um desafio perante a especificidade do enfrentamento da pandemia e a ausência de outros estudos de base populacional. Recentemente, um estudo realizado no Brasil incluindo mais de 16 mil pessoas, utilizando questionário on-line disseminado por redes sociais, identificou que $40 \%$ das pessoas respondentes estavam fazendo algum exercício durante a pandemia (sem discriminar se atividades domésticas ou de deslocamento também eram contabilizadas ${ }^{16}$. $\mathrm{O}$ percentual mais elevado comparado aos nossos resultados pode ser atribuído principalmente ao perfil de respondentes de inquéritos on-line, os quais não são representativos de todas as parcelas da população e podem também apresentar características ligadas a um maior acesso a práticas de atividade física.

Na presente análise, $24,4 \%$ das pessoas relataram a prática de atividade física de lazer em meio a pandemia e $7,7 \%$ conforme as recomendações dos órgãos de saúde. Em termos de recomendação, a OMS sugere o acúmulo de 150 minutos semanais de atividade física em quatro domínios (lazer, deslocamento, domicílio e trabalho). No entanto, o instrumento aqui proposto questionou as atividades de lazer, pelo entendimento de constituírem a principal aposta da área em termos de promoção da atividade física, tendo em vista a questão de ampliar o acesso e o gosto pelas práticas ${ }^{17}$. O período do lazer seria quando a maioria das pessoas se relacionaria às práticas como um fim em si mesmas e não como consequência impositiva de seu trabalho, atividades domésticas ou necessidade de deslocamento mais rápido ou mais barato ${ }^{18}$. Esta noção busca dialogar com a atividade física eleita pelas pessoas para enriquecer suas vidas, admitindo sentido e dignidade próprias para as práticas de lazer e, segundo o Relatório de Desenvolvimento Humano Nacional, podendo trazer contribuições para o desenvolvimento humano ${ }^{18}$.

Estudos epidemiológicos sobre a prática de atividade física oscilam em termos de instrumento, ponto de corte, população estudada e amostragem. $\mathrm{O}$ dado brasileiro mais recente indica a prevalência de 39\% de atividade física no tempo livre em adultos ${ }^{8}$. A Pesquisa Nacional de Saúde havia identificado $22,5 \%$ de adultos considerados ativos no lazer ${ }^{19}$ e ambos utilizaram o critério dos 150 minutos. Mesmo considerando as diferenças em tais estudos, é possível acionar a hipótese de que a atividade física de lazer diminuiu durante a pandemia, em função dos achados da presente pesquisa.

Destaca-se como fortaleza do presente estudo, além de seu pioneirismo já mencionado, da abordagem deste tema com dados representatividade populacional, a pactuação entre Instituições de Ensino Superior com a Secretaria de Saúde Municipal de Bagé para a produção de um conhecimento de impacto imediato para o direcionamento das políticas públicas de mitigação das consequências diretas e indiretas da pandemia de COVID-19. Como limitações, destacamos a necessidade de adaptação de questionários para aplicação no cenário atual, sem a possibilidade de testagem/validação de seus aspectos psicométricos. Além disso, ressalta-se também os desafios da coleta de dados em um contexto de pandemia, exigindo um relativo curto espaço de tempo para treinamento, além da dificuldade para a realização de estratégias de controle de qualidade. Outra limitação é a maior proporção de mulheres compondo a amostra. Assim, quando o morador sorteado no domicílio não estava presente e não podia se apresentar imediatamente para a realização da entrevista, um novo participante era sorteado, favorecendo a maior inclusão de grupos populacionais que ficavam mais tempo em casa.

Para além da descrição da prática de atividade física de lazer e reprodução do discurso de que as pessoas devam praticar atividade física no contexto da pandemia, buscamos explorar alguns aspectos socioculturais nem sempre presentes na lógica discursiva do campo da saúde. Desde o início da pandemia e do distanciamento social, a importância da atividade física foi enfatizada em textos de opinião, posicionamentos e pouco baseada em evidências cientificas. A base argumentativa indicava preocupação com a redução da atividade física no período e sustentava a ligação da prática com o sistema imune ${ }^{7}$, controle de doenças crônicas, especialmente entre os ido$\operatorname{sos}^{4}$, criação de canais de comunicação remotos entre profissionais e população ${ }^{6}$, a importância da atividade física para crianças ${ }^{20}$ e as diferenças entre as pessoas segundo prática de AF na relação com saúde mental ${ }^{21}$. Em um dos comentários, foi argumentando que algumas realidades encontrariam domicílios densos, dificuldade de acesso à internet, problemas de renda e insegurança alimentar que não fariam da atividade física uma 
prioridade $^{20}$. Esta análise é imprescindível, mas renunciada na maioria dos trabalhos. $\mathrm{O}$ que pretendemos na discussão deste artigo é ultrapassar o discurso que simplesmente destaca a importância da atividade física e retomar aspectos que interferem nesta prática.

Um dos possíveis caminhos para que a atividade física seja vista a partir de sua complexidade e não por abordagens discursivas superficiais é dar visibilidade às desigualdades, não somente pela descrição dos indicadores, mas empreendendo um olhar social e humanizado ao tema. $\mathrm{O}$ envolvimento de homens com atividade física de lazer durante a pandemia foi um pouco superior ao dobro do que das mulheres. A literatura já indicava maior atividade física de lazer em homens, mas a pandemia parece ter acentuado tal diferença. Durante o período de distanciamento social, não foram raros os debates públicos reiterando a necessidade de compartilhamento das atividades no domicílio que compreendem desde afazeres domésticos, cuidado com as demais pessoas que vivem sob o mesmo teto e a manutenção de atividades de trabalho. Embora todos deveriam se responsabilizar pelo cuidado das pessoas e da casa, na realidade parece haver uma série de barreiras sociais que comprometem as mulheres com mais atividades. As mulheres dedicam quase o dobro do tempo dos homens em tarefas domésticas ${ }^{22}$. Em meio à pandemia, uma campanha (\#ElesPorElasEmCasa) foi criada na perspectiva de enfrentar o tema e reverter a lógica dominante nas relações e consequentemente na vida e na saúde das mulheres ${ }^{23}$. As questões de gênero citadas acabam por comprometer o lazer das mulheres e consequentemente a prática de atividade física, pois caso tais práticas sejam identificadas como do interesse das mulheres, estas precisarão de tempo e condições para realizá-las. O Grupo Temático de Trabalho - Gênero do Colégio Brasileiro de Ciências do Esporte ${ }^{24}$ anunciou a preocupação com o cenário sexista, machista e paternalista em tempos de distanciamento social que acomete também as práticas corporais e de atividade física. Em uma perspectiva de integração de todos e de todas que constituem o domicílio, na desconstrução das verdades sobre os corpos, sugeriu-se danças, ginásticas e brincadeiras cantadas realizadas por homens na busca por um tensionamento nos demarcadores de gênero impostos pela nossa sociedade.

Os dados, com diferença marcante da atividade física e escolaridade, já haviam sido de- monstrados antes da pandemia, bem como o acesso ao profissional e a programas dessa área conforme indicadores sociais, sempre em favor dos mesmos grupos sociais (homens, brancos, escolarizados) $)^{8,11,19}$. Aqui novamente parece ter sido acentuada a diferença, chegando a 40 pontos percentuais de distância entre atividade física e escolaridade. A variável escolaridade, utilizada no estudo como uma aproximação da condição socioeconômica, nos ajuda a pensar as diferenças em que vivem estas pessoas e que sabidamente atinge o país. No Brasil, 5,1 milhões de domicílios (equivalente a $7,8 \%$ do total) encontram-se adensados (aglomerados subnormais), o que significa moradia em condições precárias ${ }^{25}$. São favelas, baixadas, comunidades, loteamentos e vilas onde brasileiros vivem em locais carentes de serviços essenciais como esgoto, água, energia e coleta de lixo. Compreendendo este cenário e as condições de aglomeração em tais domicílios, percebe-se que as medidas de prevenção ao SARS-CoV-2, entre elas o distanciamento social, ficam sobremaneira prejudicadas e restarão poucas condições para a prática de atividade física. Mesmo que o imperativo "faça atividade física para a saúde" chegue a ser repetido à exaustão, as condições de vida, expressas pela escolaridade ou outros indicadores, seguirão gritando e demarcando a impossibilidade de concretizar essa prática.

Isso não significa que a atividade física não possa ser promovida discursivamente e em termos de políticas públicas, o que carece às iniciativas é uma leitura crítica e atenta às múltiplas realidades e condições de vida. Durante a pandemia foi veiculado um material do Observatório de Favelas (https://www.observatoriodefavelas. org.br/) do Rio de Janeiro com áudio e imagem compartilhados em aplicativo de mensagens. O áudio dizia que "apesar dessa febre toda de dicas e pessoas falando nas lives sobre exercício físico, ninguém é obrigado nesse momento a começar nada que não queira" e "nenhum exercício físico vai te curar ou deixar imune do COVID-19". O material salientava também que sem um professor acompanhando, todo cuidado seria pouco e que se fosse fazer algo, deveria fazer o que gosta, incluindo dançar como sugestão. Tomando esta iniciativa como exemplo, sugerimos que se busque promover atividades prazerosas e que reconheçam as subjetividades e, especialmente, a dificuldade de acesso, evitando a culpabilização da vítima. 


\section{Considerações finais}

Como procuramos explicitar em nossa abordagem, a atividade física não é um gesto banal e desprovido de relação com a subjetividade das pessoas. O discurso científico-político-midiático constrói e repete a narrativa de que há necessidade de se acumular minutos de atividade física na perspectiva da saúde. Todavia, há impeditivos socioculturais que exercem centralidade na vida dos brasileiros, enquanto que o presente estudo oferece visibilidade a esse cenário ao descrever a magnitude das desigualdades na prática de ativi- dade física de lazer. Desconsiderar esse cenário complexo funcionará para a manutenção dos problemas destacados. Uma promoção de atividade física que reproduza as recomendações de saúde sem a problematização dos discursos, no sentido da humanização e da leitura da vida desigual das pessoas no Brasil, especialmente nas relações de classe, raça/etnia e gênero, pouco contribui na produção de saúde, que pode sim passar por maior envolvimento em atividades físicas, desde que contextualizadas as desigualdades que são anteriores à pandemia.

\section{Colaboradores}

DCP Pellegrini, J Harter e LP Santos conceberam o estudo maior com a colaboração de I Crochemore-Silva e BP Nunes. I Crochemore-Silva e AG Knuth conceberam o presente manuscrito, o instrumento utilizado e redigiram a primeira versão do texto. A Wendt realizou as análises estatísticas. DCP Pellegrini, J Harter, LP Santos, I Crochemore-Silva, BP Nunes, A Wendt e PC Hallal realizaram revisão crítica e aprovaram a versão final manuscrito. Os autores I Crochemore-Silva e AG Knuth compartilham primeira autoria por igual contribuição. 


\section{Referências}

1. Cucinotta D, Vanelli M. WHO Declares COVID-19 a Pandemic. Acta bio-medica: Atenei Parmensis. 2020; 91(1):157-160.

2. Anderson RM, Heesterbeek $\mathrm{H}$, Klinkenberg $\mathrm{D}$, Hollingsworth TD. How will country-based mitigation measures influence the course of the COVID-19 epidemic? Lancet 2020; 395(10228):931-934.

3. Garcia LP, Duarte E. Intervenções não farmacológicas para o enfrentamento à epidemia da COVID-19 no Brasil. Epidemiol. Serv. Saúde 2020; 29(2):e2020222

4. Sociedade Brasileira de Medicina do Exercício e do Esporte (SBMEE). Informe da Sociedade Brasileira de Medicina do Exercício e do Esporte sobre exercício físico e o coronavírus (COVID-19). 2020 [acessado 2020 Jun 15]. Disponível em: http://www.medicinadoesporte.org.br/wp-content/uploads/2020/03/sbmee_covid19_final.pdf]

5. Brasil. Ministério da Saúde (MS). Como fica a prática de atividade física durante a pandemia de Coronavírus? 2020 [acessado 2020 Jun 15]. Disponível em: https://saudebrasil.saude.gov.br/eu-quero-me-exercitar-mais/como-fica-a-pratica-de-atividade-fisica-durante-a-pandemia-de-coronavirus

6. Souza Filho BAB, Tritany EF. COVID-19: importância das novas tecnologias para a prática de atividades físicas como estratégia de Saúde Pública. Cad Saude Publica 2020; 36(5):e00054420.

7. Chen P, Mao L, Nassis GP, Harmaer P, Ainsworth BE, Li F. Coronavirus disease (COVID-19):The need to maintain regular physical activity while taking precautions. J Sport Health Sci 2020; 9(2):103-104.

8. Brasil. Ministério da Saúde (MS). Vigitel Brasil 2019: vigilância de fatores de risco e proteção para doenças crônicas por inquérito telefônico. Brasília: MS; 2020.

9. Brasil. Ministério da Saúde (MS). Vigitel Brasil 2006: vigilância de fatores de risco e proteção para doenças crônicas por inquérito telefônico. Brasília: MS; 2007.

10. Ferreira RW, Caputo EL, Häfele CA, Santos JS, Florindo A, Knuth AG, Silva MC. Acesso aos programas públicos de atividade física no Brasil: Pesquisa Nacional de Saúde, 2013. Cad Saude Publica 2019; 35(2):e00008618.

11. Oliz MM, Dumith SC, Knuth AG. Utilização de serviços de educação física por adultos e idosos no extremo sul do Brasil: estudo de base populacional. Cien Saude Colet 2020; 25(2):541-552.

12. Minayo M, Freire N. Pandemia exacerba desigualdades na Saúde. 2020. [acessado 2020 Jun 15]. Disponível em: https://www.abrasco.org.br/site/noticias/pandemia-exacerba-desigualdades-na-saude-artigo/48293

13. Hallal PC, Horta BL, Barros AJD, Dellagostin OA, Hartwig FP, Pellanda LC, Struchiner CJ, Burattini MN Silveira MF, Menezes AMB, Barros FC, Victora CG. Trends in the prevalence of COVID-19 infection in Rio Grande do Sul, Brazil: repeated serological surveys. Cien Saude Colet 2020; 25(Supl. 1):2395-401.

14. Estatística I-IBdGe. IBGE Cidades: Bagé. 2020 [acessado 2020 Jun 15]. Disponível em: https://cidades.ibge. gov.br/brasil/rs/bage/panorama

15. World Health Organization (WHO). Global recommendations on physical activity for health. Geneva: WHO; 2010.
16. Bezerra ACV, Silva C, Soares FRG, Silva JAM. Factors associated with people's behavior in social isolation during the COVID-19 pandemic. Cien Saude Colet 2020; 25(Supl. 1):2411-2421.

17. Crochemore-Silva I, Knuth AG, Mielke GI, Loch MR. Promotion of physical activity and public policies to tackle inequalities: considerarions based on the Inverse Care Law and Inverse Equity Hypothesis. Cad Saude Publica 2020; 36(6):e00155119.

18. Programa das Nações Unidas para o Desenvolvimento (PNUD). Relatório Nacional de Desenvolvimento Humano do Brasil. Movimento é vida: atividades físicas e esportivas para todas pessoas. Brasília: PNUD; 2017.

19. Mielke GI, Hallal PC, Rodrigues GBA, Szwarcwald CL, Santos FV, Malta DCl. Prática de atividade física e hábito de assistir à televisão entre adultos no Brasil: Pesquisa Nacional de Saúde 2013. Epidemiol. Serv. Saúde 2015; 24(2):277-286.

20. Guan H, Okely AD, Aguilar-Farias N, Cruz BP, Draper CE, Hamdouchi AE, Florindo AA, Jauregui A, Katzmarzyk PT, Kontsevaya A, Lof M, Parl W, Reilly JJ, Sharma D, Tremblay MS, Veldman SLC. Promoting healthy movement behaviours among children during the COVID-19 pandemic. Lancet Child Adolesc Health 2020; 4(6):416-418.

21. Lesser IA, Nienhuis CP. The Impact of COVID-19 on Physical Activity Behavior and Well-Being of Canadians. Int. J. Environ. Res. Public Health 2020; 17(11):3899

22. Instituto Brasileiro de Geografia e Estatística (IBGE). Coordenação de Trabalho e Rendimento. PNAD contínua: outras formas de trabalho. Rio de Janeiro: IBGE; 2018.

23. \#ElesPorElasEmCasa incentiva homens brasileiros a mostrar tarefas domésticas assumidas na quarentena da Covid-19 2020. [acessado 2020 Jun 15]. Disponível em: http://www.onumulheres.org.br/noticias/ elesporelasemcasa-incentiva-homens-brasileiros-amostrar-tarefas-domesticas-assumidas-na-quarentena-da-covid-19/

24. Grupos de Trabalhos Temáticos do Colégio Brasileiro de Ciências do Esporte (CBCE):Gênero. Práticas Corporais em tempos de distanciamento social e coronavírus Nota Técnica 6 - GTT Gênero do CBCE, 2020. [acessado 2020 Jun 15]. Disponível em: http://www. cbce.org.br/upload/biblioteca/GTT\%20G\%C3\%AAnero $\% 20-\% 20$ CBCE $\% 20 \mathrm{em} \% 20$ tempos $\% 20 \mathrm{de} \% 20$ coronav\%C3\%ADrus.pdf

25. Instituto Brasileiro de Geografia e Estatística (IBGE). Aglomerados Subnormais. 2019. [acessado 2020 Jun 15]. Disponível em: https://socecodem-ibgedgc.hub. arcgis.com/app/ac337eeee5164c0daa9c99f8689ad3f8

Artigo apresentado em 21/06/2020

Aprovado em 10/09/2020

Versão final apresentada em 12/09/2020 